\title{
Reverse Brazil Nut Problem: Competition between Percolation and Condensation
}

\author{
Daniel C. Hong and Paul V. Quinn \\ Physics, Lewis Laboratory, Lehigh University, Bethlehem, Pennsylvania 18015 \\ Stefan Luding \\ Institut für Computeranwendungen 1 Pfaffenwaldring 27, 70569 Stuttgart, Germany
}

\begin{abstract}
In the Brazil nut problem (BNP), hard spheres with larger diameters rise to the top. There are various explanations (percolation, reorganization, convection), but a broad understanding or control of this effect is by no means achieved. A theory is presented for the crossover from BNP to the reverse Brazil nut problem (RBNP) based on a competition between the percolation effect and the condensation of hard spheres. The crossover condition is determined, and theoretical predictions are compared to Molecular Dynamics simulations in two and three dimensions.
\end{abstract}

Rosato et al. [1] demonstrated via Molecular Dynamics (MD) simulations that hard spheres with large diameters segregate to the top when subjected to vibrations or shaking. In the literature, this phenomenon is called the Brazil nut problem (BNP) [2]. Besides a broad experience in the applied and engineering sciences $[2,3]$, there exist more recent approaches to understanding this effect through model experiments [4-7]. The BNP has been attributed to the following phenomena: the percolation effect, where the smaller ones pass through the holes created by the larger ones [2], geometrical reorganization, through which small particles readily fill small openings below the large particles $[3,5]$ and global convection which brings the large particles up but does not allow for re-entry in the downstream [4]. Since most experiments were carried out with a single large grain in a sea of smaller ones [4-6], it is not quite clear which of these observed mechanisms apply for the segregation of binary mixtures. For example, while the convection is responsible for the rise of the single large grain, MD simulations [8] and hydrodynamic models [9] clearly indicate that convection cells are absent in the bulk, but confined near the wall when the width of the container is much larger than 
the height. Recently, Shinbrot and Muzzio [10] observed a reverse buoyancy in shaken granular beds, where again a single large grain in a sea of smaller grains could segregate to the bottom if the bed was deep and the amplitude of vibration was large. While all these scenarios are interesting, we recognize that the basic control parameters for each regime have not been clearly identified. Numerical simulations of binary mixtures with external temperature gradients and varying ratios of gravitational acceleration and agitation intensity [11] show that segregation can be tuned, avoided and even inversed under special, but non-practical boundary conditions. However, these simulations did not examine a variation in mass-ratio or size-ratio and were not compared to the kinetic theory of binary mixtures [12].

The purpose of this Letter is to propose a new condensation driven segregation of binary hard spheres under gravity, which is conceptually different from the previously known convection driven [4], percolation driven [2,3], reorganization controlled [5], entropy driven (in the absence of gravity) [7], or inertia driven [10] segregation processes. We will identify the control parameter(s) and determine the crossover condition from the normal BNP to the reverse Brazil nut problem (RBNP).

The starting point is the observation made in an earlier paper [13] and recently confirmed by MD simulations [14] that there exists a critical temperature, $T_{c}$, below which a monodisperse system of hard spheres undergoes a condensation transition in the presence of gravity. The density profile was obtained using the global equation of state of the system [15]. Consider, for example, a system of elastic hard spheres with mass $m$ and diameter $d$ in a container. Let the initial layer thickness (filling height at rest when $T=0$ ) be $\mu$ measured in units of $d$. If the system is in contact with a thermal reservoir at temperature $T$, one can estimate the dimensionless thickness of the fluidized layer, $\Delta h$, in $\mathrm{D}$ dimension by equating the kinetic energy of one grain to the potential energy equivalent, $m\left\langle v^{2}\right\rangle / 2=D T / 2 \approx m g d \Delta h$, which yields $\Delta h \approx D T /(2 m g d)$. One may estimate the point, $T_{c}$, at which the system is fully fluidized, by setting $\Delta h \approx \mu$. We obtain:

$$
T_{c}=m g d \mu / \mu_{o},
$$

where $\mu_{o}$ is a constant that depends on the spatial dimension and the underlying packing structure [16]. $\mu_{o}$ was determined in Ref. [13] with the use of the Enskog pressure, while the numerically determined values for $\mu_{o}$ were provided in Ref. [14]. 
When $T>T_{c}$, the system is fully fluidized and the dimensionless thickness of the solid, defined as $h_{\text {solid }}(T)=\mu-\Delta h(T)$, vanishes. On the other hand, for $T<T_{c}$, one finds $h_{\text {solid }} \neq 0$ because a fraction of particles condenses at the bottom. Here, the solid phase refers to a hard sphere state where each particle fluctuates around a fixed point, but is confined in a cage so that it can not exchange its center of mass position with neighboring particles [14].

Consider now a binary mixture of hard spheres with species $\mathrm{A}$ and $\mathrm{B}$ having mass $m_{A}$ and $m_{B}$ and diameters $d_{A}$ and $d_{B}$, respectively, and the initial layer thicknesses are $\mu_{A}$ and $\mu_{B}$. From Eq. (1), we find the ratio of the critical temperatures to be

$$
\frac{T_{c}(A)}{T_{c}(B)}=\frac{m_{A} d_{A}}{m_{B} d_{B}},
$$

where we have assumed that $\mu_{A}=\mu_{B}$. Suppose the system is quenched at a temperature $T$ between the two critical temperatures $T_{c}(A)$ and $T_{c}(B)$, i.e. $T_{c}(B)<T<T_{c}(A)$. Then, the hard spheres of type $\mathrm{B}$ are above the condensation temperature, while the hard spheres of type A are below it. Thus at temperature $T$, particles of type A will try to condense, while particles of type B remain fluidized. Hence, type A particles will tend to segregate to the bottom first. The underlying assumption of this scenario is that particles of type A only interact with themselves while seeing particles of type B as phantom particles, and vice versa. Even though this assumption is a crude one, we nevertheless find that the prediction based on this assumption appears to work well for the segregation of binary mixtures under the influence of gravity.

Since the critical temperature depends on $m$ and $d$, an inversion of the segregation should be achieved by simply altering the values of $m$ and $d$. We first present Event-Driven Molecular Dynamics data for the normal Brazil nut problem(BNP), where particles with a larger diameter rise to the surface. Subsequently, we demonstrate how to reverse this phenomenon by quenching the system between the two condensation temperatures given by Eq. (1) and used in Eq. (2).

The total number of particles used in our simulations varied from 400 to 2,000 in $2 \mathrm{D}$, and 2000 to 3,600 in $3 \mathrm{D}$, with the width of the container varying from 15 to 50 particle diameters in 2D, and staying fixed at $15 \times 15$ in 3D. We refer the readers to references [17] for details of the algorithm, which takes into account the rotation, regarding the collisional dynamics of hard spheres and a method for handling the inelastic collapse. The thermal reservoir of our 

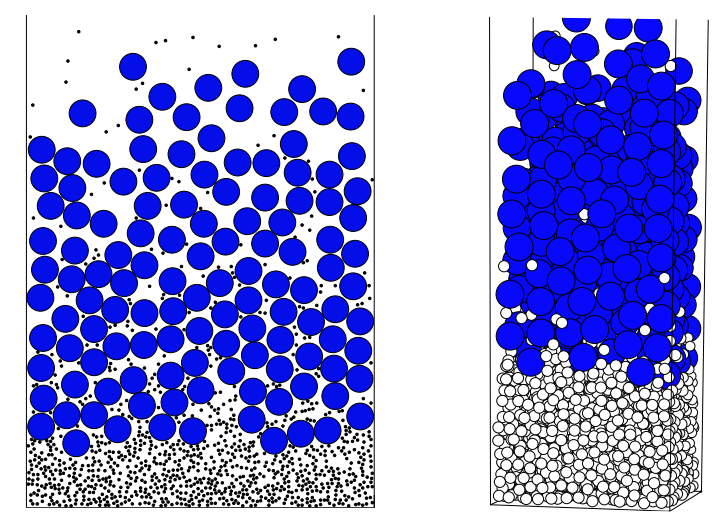

Figure 1: Brazil nut problem in 2D (a) and 3D (b). Particles with the larger diameter rise to the top. Note that $T_{c}(B)<T<T_{c}(A)$ and percolation overrides the condensation effect.

system was modeled using white noise driving, first introduced by Williams and MacKintosh [18]. The strength of the noise was adjusted such that the average kinetic energy of all particle defines the kinetic temperature of the system.

To test the theory properly, the system was started from a completely mixed state with no bias. To accomplish this, the binary mixture was first heated to a very high temperature with no gravity in a closed container. This allowed the system to reach a proper steady state where the centers of mass of both species A and B were the same, and the density profiles of both species were uniform throughout the container. Under these conditions, we achieved a perfectly mixed binary system. We then turned on gravity, removed the lid of the container, and quenched the system to a temperature, $T$, between the two critical temperatures $T_{c}(A)$ and $T_{c}(B)$, such that $T_{c}(B)<T_{c}(A)$. The system was then allowed to relax to steady state under these new conditions. Then we measured the density profile, and monitored the center of mass. The coefficient of restitution was 0.9999 for the particles and 0.98 for the walls, values where the system did not suffer from inelastic collapse [19]. We first present MD data for the usual Brazil nut problem [1]. Such a case is shown in Fig. 1 for $d_{A} / d_{B}=8$, and $m_{A} / m_{B}=4$ in $2 \mathrm{D}$ and $d_{A} / d_{B}=2$, and $m_{A} / m_{B}=2$ in $3 \mathrm{D}$. The transition temperature ratio is $T_{c}(A) / T_{c}(B)=$ $m_{A} d_{A} / m_{B} d_{B}=8$ in $2 \mathrm{D}$ and 4 in $3 \mathrm{D}$, and the quenching temperature $T$ is such that $T_{c}(B)<T<T_{c}(A)$. In these cases, we expect the larger particles 


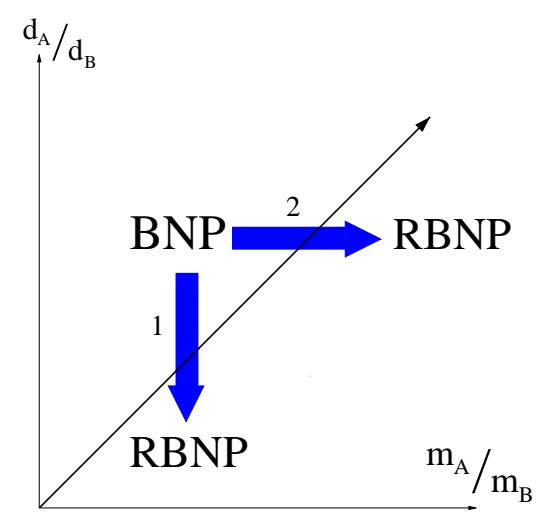

Figure 2: The schematic picture for the crossover from the Brazil Nut Problem (BNP) to the Reverse Brazil Nut Problem (RBNP). The line is the dividing line between the BNP and the RBNP for $2 \mathrm{D}$.

to condense first, while the smaller particles remain fluidized. Indeed, the center of mass position of particles of type A quickly decays as predicted by the condensation picture, but as time passes, the smaller particles of type B evantually pass through the holes and settle to the bottom because of the percolation effect. Percolation overrides the condensation mechanism, resulting in the usual BNP in two and three dimensions. Snap shots of the equilibrium state, which is the typical Brazil nut segregation [1,5,7], are shown in Fig. 1. At this point, one has two choices leading to the RBNP, the schematic picture of which is shown in Fig. 2. The first option is to fix the mass ratio and change the diameter ratio (Path 1 ). There exists a critical diameter ratio below which the RBNP sets in. This was also observed in Ref. [6]. The second case is to fix the diameter ratio and change the mass ratio.(Path 2) Again, there exists a critical mass ratio beyond which the RBNP sets in. Both cases exhibit a crossover from the percolation to the condensation driven segregation. We now present representative MD data to demonstrate this crossover from BNP to RBNP. We changed both the mass and the diameter, keeping $\mu_{A}=\mu_{B}$, to reverse the Brazil nut segregation via condensation. In Fig. 3a, simulation results are shown for the same mass ratio as Fig. 1a, i.e $m_{A} / m_{B}=4$, but a diameter ratio of $d_{A} / d_{B}=2$. This represents movement along path 1 in the parameter space of Fig. 2. We quenched the system between the two critical temperatures such that $T_{c}(B)<T<T_{c}(A)$. In this case, the larger particles condensed first and 

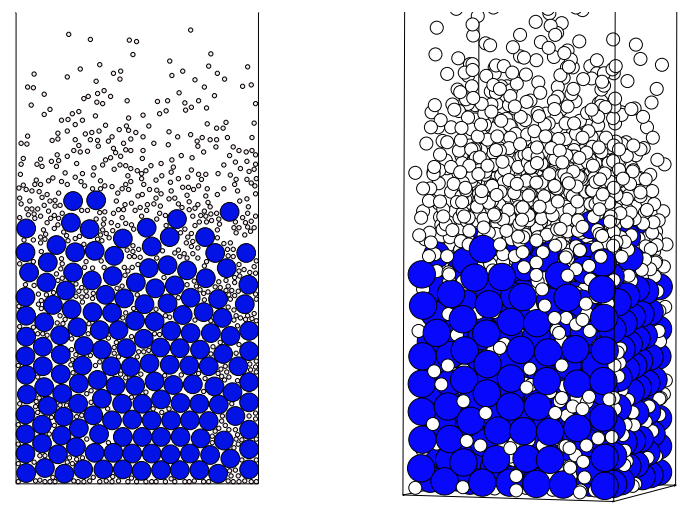

Figure 3: (a) RBNP along path 1 in 2D. Particles with the larger diameter sink to the bottom. The mass ratio is the same as in Fig. 1a, but the diameter ratio decreased to $d_{A} / d_{B}=2$. (b) RNBP along path 2 in 3D. The diameter ratio is the same as in Fig. $1 \mathrm{~b}$, but the mass ratio is increased to $m_{A} / m_{B}=6$

settled to the bottom. They overcame the percolation effect and remained there, even though the diameter was larger. In Fig. 3a, the simulation results clearly show the RBNP, namely, the larger ones settling to the bottom.

One may also observe the RBNP by moving down along path 2 as shown in Fig. 2. The corresponding simulation result for the RBNP is shown in Fig. $3 \mathrm{~b}$, where the diameter ratio is $d_{A} / d_{B}=2$, but the mass ratio is $m_{A} / m_{B}=6$.

The simulation results clearly suggest that during the segregation process, both the percolation effect and the condensation mechanism compete. Hence, the condition for the crossover from the BNP to the RBNP may be determined by setting the control parameters for both cases to be of the same order of magnitude.

The control parameter for the percolation effect has been determined by Rosato et al. [1], who argued that it is basically controlled by the ratio of volumes (diameters) of the two types of particles. Since we have established that the dimensionless control parameter for condensation is the ratio of the two critical temperatures, we determine the crossover condition as follows:

$$
\left(\frac{d_{A}}{d_{B}}\right)^{D} \approx \frac{T_{c}(A)}{T_{c}(B)}=\frac{m_{A} d_{A}}{m_{B} d_{B}},
$$



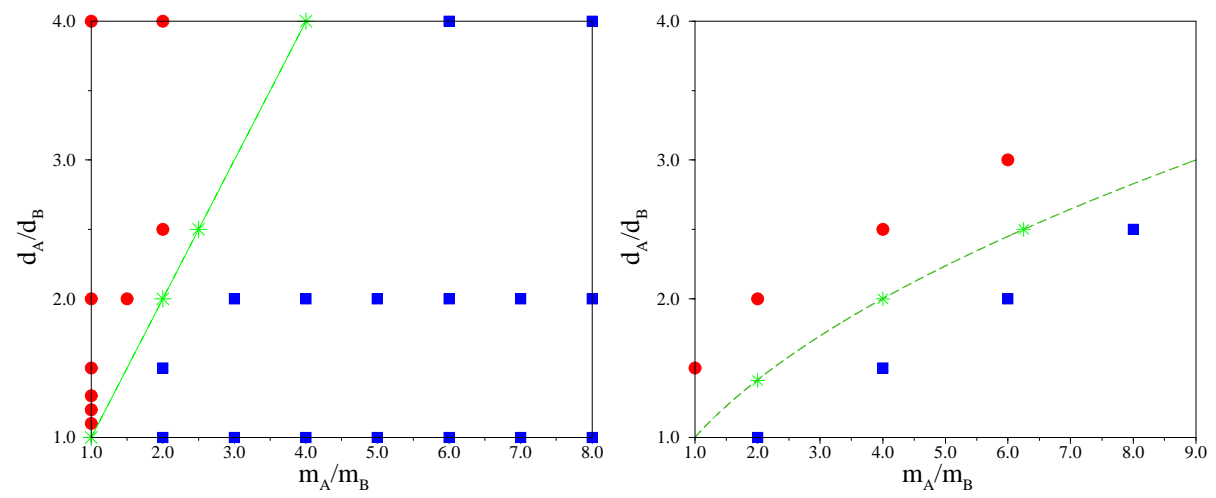

Figure 4: Phase diagram determined for the segregation of binary mixtures in (a) two dimensions, and (b) three dimensions. In 2D, the straight line is $y=x$, while in $3 \mathrm{D}$, the curve is $y=\sqrt{x}$. The circles are data points for BNP, the squares for RBNP, and the stars are data points for the mixed state.

or equivalently,

$$
\left(\frac{d_{A}}{d_{B}}\right)^{D-1} \approx \frac{m_{A}}{m_{B}},
$$

where $D$ is the spatial dimension. The simulation results are displayed in Fig. 4a for two dimensions and Fig. 4b for three dimensions.

The vertical axis is $y=d_{A} / d_{B}$ and the horizontal axis is $x=m_{A} / m_{B}$. Boundaries for the crossover from the BNP to RBNP are given by the curve, $y=x^{1 /(D-1)}$, where $y=x$ in $2 \mathrm{D}$ and $y=\sqrt{x}$ in 3D. At the boundary line (stars), the system does not really segregate, but remains in a mixed state. In passing, we point out that we have also investigated the effect of the layer thickness on segregation. If we make $\mu_{A}>\mu_{B}$, such that $T_{c}(B)<T<T_{c}(A)$, then we can destroy the BNP such that a mixed state occurs.

In summary, we have proposed a qualitative model for the segregation of binary mixtures and demonstrated that the type of segregation (BNP or RBNP) can be predicted. The range of applications is beyond that of the classical percolation, reorganization, arching, and convection models since those are limited to special situations. For example, the arching model requires very small temperatures or rather high densities in the condensed regime, whereas the convection model can only be applied if convection cells are present. However, we do not exclude those approaches. Rather, we 
expect that our condensation driven segregation gives a more general framework, while special cases can be described better using the adapted models mentioned above. We have related the segregation to the respective condensation of two species, which takes place at different temperatures, and have studied various mass and size ratios confirming the theoretical predictions via MD simulations in both two and three dimensions. The future research needs to focus on the effect of the filling heights on the condensation driven segregation and examining our strong assumption that the particles of either species feel the other species only as phantom background.

We wish to thank T. Shinbrot and Yan Levin for comments on the manuscript. S. L. acknowledges the support of the Deutsche Forschungsgemeinschaft (DFG).

[1] T. Rosato et al., Phys. Rev. Lett. 58, 1038 (1987).

[2] W. Reisner and E. Rothe, in Bins and Bunkers for Handling Bulk Materials, series on Rock and Soil Mechanics (Trans Tech, Clausthal-Zellerfeld, West Germany, 1971); Z. T. Chowhan, Pharm. Technol. 19, 56 (1995); S. Luding et al., Pharm. Technol. 20, 42 (1996).

[3] J. C. Williams, Powder Technol. 15, 245 (1976); J. Bridgwater, Powder Technol. 15, 215 (1976); W. M. Visscher and M. Bolsterli, Nature(London) 239, 504 (1972).

[4] J. Knight, H. Jaeger and S. Nagel, Phys. Rev. Lett. 70, 3728 (1993).

[5] J. Duran and J. Rajchenbach and E. Clément, Phys. Rev. Lett. 70, 2431 (1993); W. Cooke et al., Phys. Rev. E 53, 2812 (1996).

[6] R. Julien, P. Meakin, and A. Pavlovitvh, Phys. Rev. Lett. 69, 640 (1992).

[7] M. Dijikstra and D. Frenkel, Phys. Rev. Lett. 72, 298 (1994).

[8] Y-h. Taguchi, Phys. Rev. Lett. 69, 1367 (1992); See also, Int. J. Mod. Phys. A. 7, 1839 (1993).

[9] D. C. Hong and S. Yue, Phys. Rev. E. 58, 4763 (1998).

[10] Shinbrot and Muzzio, Phys. Rev. Lett. 81, 4365 (1998).

[11] S. McNamara and S. Luding, in: Segregation in Granular Flows, ed. T. Rosato, IUTAM Symposium Proc. (Kluwer, Dordrecht, 2000); S. Luding, O. Strauß and S. McNamara, ibid.

[12] J. T. Willits and B. Ö. Arnarson, Phys. of Fluids 11, 3116 (1999).

[13] D. C. Hong, Physica A 271, 192 (1999).

[14] P. V. Quinn and D. C. Hong, cond-mat/0005196. 
[15] S. Luding and O. Strauß, in Granular Gases, T. Pöschel and S. Luding, eds., Springer Verlag, Berlin (2000); S. Luding, Europhys. Lett. (submitted). [16] J. P. Hansen and I. R. McDonald, Theory of simple liquids, Academic Press Limited, London (1986).

[17] B. D. Lubachevsky, J. Comp. Phys. 94, 255(1991); S. Luding, Phys. Rev. E 52, 4442 (1995); S. Luding and S. McNamara, Granular Matter $\mathbf{1}(3), 113$ (1998).

[18] D. M. Williams and F. C. MacKintosh, Phys. Rev. E 54, R9 (1996).

[19] I. Goldhirsch and G. Zanetti, Phys. Rev. Lett. 70, 1619 (1993); S. McNamara and W. R. Young, Phys. Rev. E 53, 5089 (1996). 


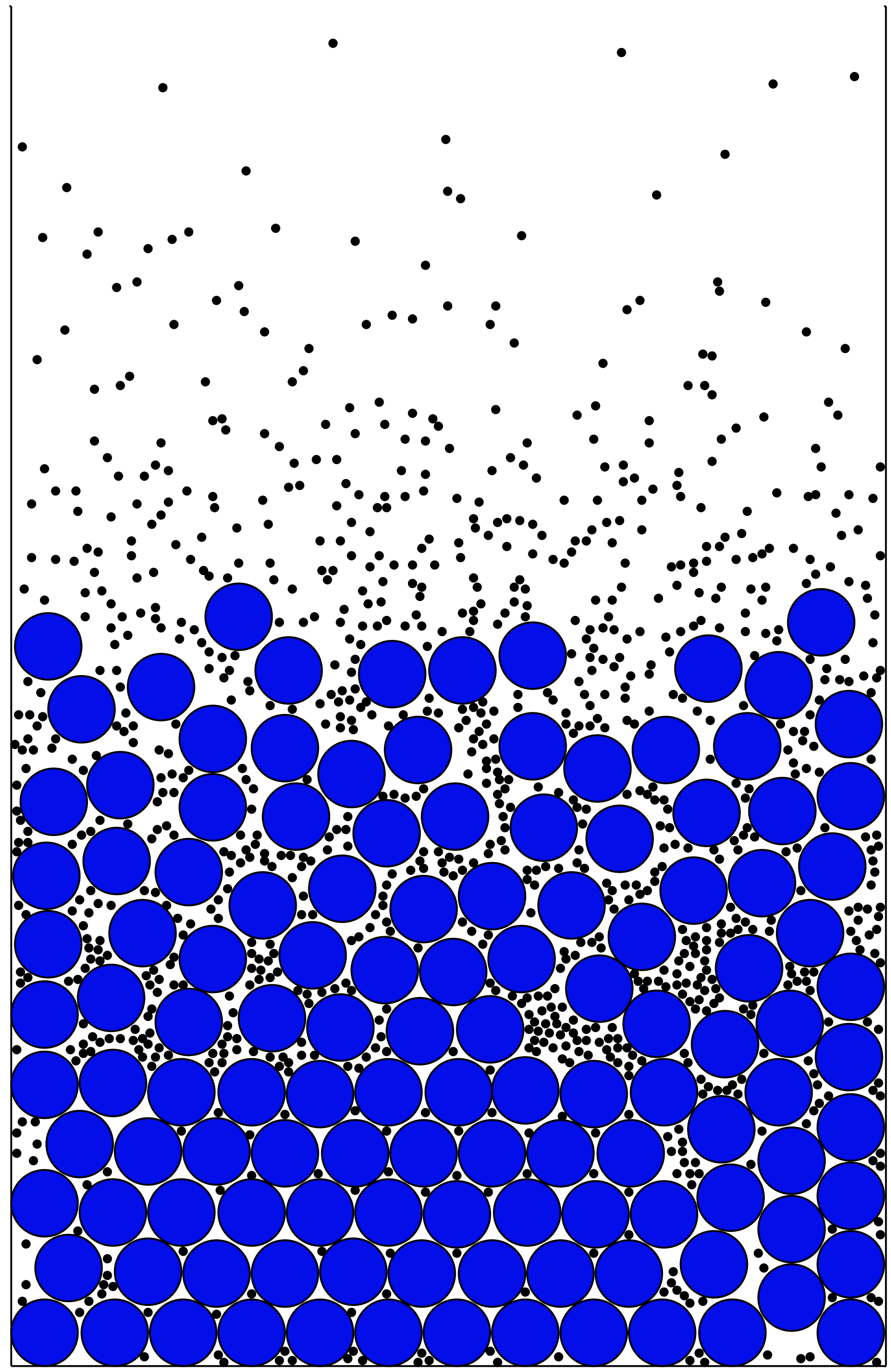

